\title{
Toxicidade de compostos nitrogenados em peixes influenciada por parâmetros físico- químicos da água: uma revisão narrativa
}

\author{
Toxicity of nitrogen compounds in fish influenced by physico-chemical water parameters: a review \\ Toxicidad de compuestos de nitrógeno em peces influenciada por parâmetros físico-químicos: uma
} revisión narrativa

Recebido: 24/08/2021 | Revisado: 29/08/2021 | Aceito: 31/08/2021 | Publicado: 04/09/2021

Thaís Paula de Araújo

ORCID: https://orcid.org/0000-0001-8672-5325 Universidade Federal de São João del-Rei, Brasil E-mail: thaisp_araujo@ outlook.com

Ludmila Silva Brighenti ORCID: https://orcid.org/0000-0003-1305-2689 Universidade do Estado de Minas Gerais, Brasil E-mail: ludmila.brighenti@uemg.br

Hélio Batista dos Santos ORCID: https://orcid.org/0000-0001-6813-8522 Universidade Federal de São João del-Rei, Brasil E-mail: hbsufsj@gmail.com

Ana Hortência Fonseca Castro ORCID: https://orcid.org/0000-0003-3155-3241 Universidade Federal de São João del-Rei, Brasil E-mail: acastro@ufsj.edu.br Ralph Gruppi Thomé ORCID: https://orcid.org/0000-0002-1779-5036 Universidade Federal de São João del-Rei, Brasil E-mail: ralph@ufsj.edu.br

\begin{abstract}
Resumo
Os compostos nitrogenados (nitrito, nitrato e amônia) são considerados tóxicos para os peixes, pois são capazes de promover alterações histológicas e bioquímicas, diminuir a capacidade de trasnporte de oxigênio no sangue e afetar o fitness (i.e. aptidão do organismo em termos de crescimento e natação). Esses compostos acumulam no ambiente aquático através da excreção de organismos aquáticos, da decomposição de matéria orgânica tanto de origem aquática quanto do sistema terrestre adjacente, e da entrada direta pelo sistema terrestre, afluentes e águas subterrâneas. As chamadas mudanças globais, como as climáticas e de uso do solo, podem potencializar e intensificar essa entrada dos compostos nitrogenados através de atividades humanas, tais como a agricultura e o lançamento de efluentes, como esgoto doméstico. Os peixes estão comumente em contato com diversos poluentes, e a combinação de fatores ambientais como temperatura, $\mathrm{pH}$, disponibilidade de oxigênio e dureza podem ter efeitos sinérgicos ou antagônicos. No entanto, como esses fatores ambientais afetam a toxicidade da amônia, nitrito e nitrato não são totalmente compreendidos. Portanto, nosso objetivo foi revisar a literatura afim de abordar os efeitos dos compostos nitrogenados, e como os parâmetros físico-químicos da água afetam a toxicidade desses compostos.
\end{abstract}

Palavras-chave: Antrópico; Ciclo do nitrogênio; Histopatologias; Impacto; Poluentes.

\begin{abstract}
Nitrogen compounds (nitrite, nitrate and ammonia) are considered toxic to fish, as they can promote histological and biochemical changes, decrease the oxygen-carrying capacity in the blood and affect fitness (ie the body's fitness in terms of growth and swimming). These compounds accumulate in the aquatic environment through the excretion of aquatic organisms, decomposition of organic matter from both aquatic and adjacent terrestrial systems, and direct entry into the terrestrial system, tributaries and groundwater. The so-called global changes, such as climate and landuse changes, can enhance and intensify this entry of nitrogen compounds through human activities, such as agriculture and the release of effluents, such as domestic sewage. Fish are commonly in contact with various pollutants, and the combination of environmental factors such as temperature, $\mathrm{pH}$, oxygen availability and hardness can have synergistic or antagonistic effects. However, how these environmental factors affect the toxicity of ammonia, nitrite and nitrate are not fully understood. Therefore, our objective was to review the literature to address the effects of nitrogen compounds, and how the physicochemical parameters of water affect the toxicity of these compounds.
\end{abstract}

Keywords: Anthropic; Nitrogen cycle; Histopathologies; Impact; Pollutants. 


\begin{abstract}
Resumen
Los compuestos de nitrógeno (nitrito, nitrato y amoníaco) se consideran tóxicos para los peces, ya que pueden promover cambios histológicos y bioquímicos, disminuir la capacidad de transporte de oxígeno en la sangre y afectar la aptitud (es decir, la aptitud del cuerpo en términos de crecimiento y natación). Estos compuestos se acumulan en el medio acuático a través de la excreción de organismos acuáticos, la descomposición de materia orgánica de los sistemas acuáticos y terrestres adyacentes y la entrada directa al sistema terrestre, afluentes y águas subterráneas. Los llamados cambios globales, como los cambios climáticos y de uso de la tierra, pueden mejorar e intensificar esta entrada de compuestos nitrogenados a través de actividades humanas, como la agricultura y la liberación de efluentes, como las águas residuales domésticas. Los peces suelen estar en contacto con diversos contaminantes y la combinación de factores ambientales como la temperatura, el pH, la disponibilidad de oxígeno y la dureza puede tener efectos sinérgicos o antagónicos. Sin embargo, no se comprende completamente cómo estos factores ambientales afectan la toxicidad del amoníaco, nitrito y nitrato. Por lo tanto, nuestro objetivo fue revisar la literatura para abordar los efectos de los compuestos nitrogenados y cómo los parámetros fisicoquímicos del água afectan la toxicidad de estos compuestos.
\end{abstract}

Palabras clave: Antrópico; Ciclo del nitrógeno; Histopatologías; Impacto; Contaminantes.

\title{
1. Introdução
}

As atividades antrópicas vêm modificando o meio ambiente de diferentes formas, e alterações no ciclo do nitrogênio estão entre as principais causas da perda de espécies. De fato, os compostos nitrogenados (nitrito, nitrato e amônia) podem ser encontrados naturalmente nos ecossistemas aquáticos, através da decomposição e excreção de alguns organismos, porém precisamos nos atentar a intensificação desta entrada por atividades antrópicas, como atividades industriais e uso de fertilizantes na agricultura (Randall \& Tsui, 2002). Neste sentido, o aumento de compostos nitrogenados na água é geralmente associado a mudança no uso do solo devido à conversão de áreas de mata nativa em áreas de agricultura e pecuária (Vitousek, 1997). Além disso, o aumento da concentração dos compostos nitrogenados também pode ser ocasionado pela perda de volume de água associada a grandes períodos de seca com relação as mudanças climáticas (Wang et al., 2020).

Dentre os compostos nitrogenados analisados nesta revisão, a amônia é um dos poluentes mais preocupantes em ambientes aquáticos naturais e da piscicultura intensiva (Martinez et al., 2006). A amônia constitui principal produto de excreção dos peixes e no ambiente é encontrada como nitrogênio amoniacal total (NAT) $\left(\mathrm{NH}_{3}-\mathrm{N}\right)$, que atravessa livremente a membrana celular, sendo, portanto, considerada potencialmente tóxica (Mehta, 2017). O nitrito é encontrado naturalmente nos organismos e também é produzido pela oxidação de íons amônio, através do processo de nitrificação (Ha et al., 2019). O nitrato é tóxico devido a conversão endógena de nitrato em nitrito, causando a formação de metahemoglobina, que resulta na perda da capacidade de transporte de oxigênio no sangue (Gomez Isaza et al., 2021).

A toxicidade dos poluentes pode variar de acordo com a concentração, condições da qualidade de água, período de exposição e sensibilidade da espécie (Kim \& Kang, 2016; Kubitza, 2019). Os organismos aquáticos estão em contato direto com diversos agentes tóxicos, e são frequentemente expostos a flutuações em parâmetros que refletem a qualidade da água, como oxigênio dissolvido, temperatura, pH, salinidade e dureza (Zhao et al., 2020). A exposição simultânea a estressores ambientais pode ter efeitos sinérgicos (i.e. quando o efeito de dois ou mais estressores é maior do que o efeito do estressor individual) ou antagonistas (i.e. quando o efeito de dois ou mais estressores é menor que o individual) (Côté et al., 2016). Além disso, o aumento da temperatura tem impacto na fisiologia dos peixes (Alfonso et al., 2020) e pode influenciar na toxicidade dos compostos nitrogenados (Mehta, 2017).

As alterações nos parâmetros ambientais aquáticos podem ser provocadas pelas mudanças globais, e ainda não sabemos claramente como esses fatores ambientais de qualidade da água afetam fisiologicamente as diferentes espécies de peixes. Portanto, nosso objetivo foi revisar a literatura afim de abordar os efeitos dos compostos nitrogenados (nitrito, nitrato e amônia) sob diferentes condições dos parâmetros físico-químicos da água, e assim avaliar como esses parâmetros influenciam na toxicidade do nitrito, nitrato e amônia. 


\section{Metodologia}

\subsection{Estratégias de busca}

As buscas dos estudos foram realizadas nas bases de dados: Google Scholar, PubMed e ScienceDirect, seguindo a metodologia proposta por Ferrari (2015). Os descritores utilizados foram: "toxicity nitrate fish", "toxicity nitrite fish", "toxicity ammonia fish".

\subsection{Critérios de inclusão/exclusão}

Apenas artigos originais de 2017 a 2021 foram analisados. Os critérios de inclusão foram: peixes de água doce e salgada, estudos envolvendo um ou mais compostos nitrogenados (amônia, nitrito ou nitrato) e influência de algum parâmetro de qualidade da água (temperatura, $\mathrm{pH}$, disponibilidade de oxigênio dissolvido, salinidade ou dureza). Os critérios de exclusão foram: estudos com associação a outros agentes tóxicos. O processo de seleção dos artigos está descrito na Figura 1.

Figura 1. Fluxograma do processo de seleção dos artigos.

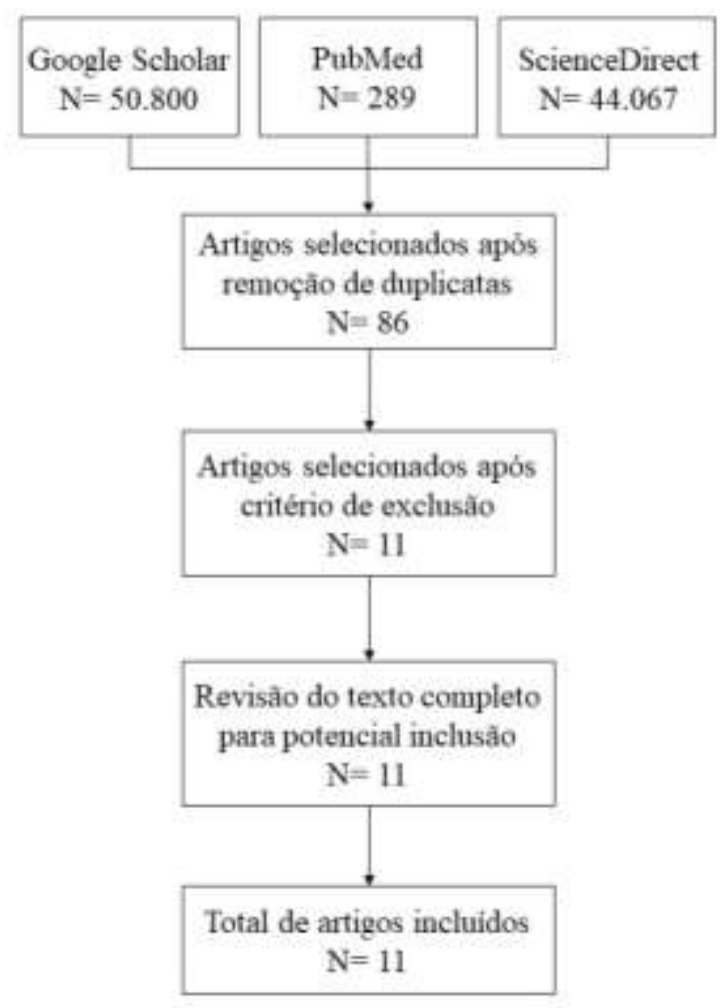

Fonte: Autores (2021).

\section{Resultados e Discussão}

\subsection{Efeitos da amônia e fatores ambientais que influenciam na sua toxicidade}

A amônia é um potencial poluente, pois pode afetar severamente a homeostase de organismos como os peixes. Estudos avaliando a toxicidade da amônia retratam que a amônia induz o estresse oxidativo, alterando a atividade de enzimas antioxidantes (Kim et al., 2017; Zhang et al., 2019), bem como induz a imunossupressão, inflamação e apoptose (Li et al., 2020). Além disso, a amônia é capaz de causar histopatologias associadas as brânquias e ao fígado, como deslocamento do epitélio, hiperemia (aumento do fluxo sanguíneo) e necrose nas brânquias, e danos no tecido hepático, como vacuolização e inchaço dos hepatócitos (Silva et al., 2017). 
Dados de literatura mostram que a elevação da temperatura da água pode induzir o estresse e diminuir o crescimento dos peixes (Lefevre et al., 2017; Pankhurst \& Munday, 2011). Avaliando-se conjuntamente o aumento de temperatura e o acúmulo de amônia na água, é possível notar que a temperatura é capaz de potencializar a toxicidade da amônia em peixes juvenis. Kim e colaboradores (2017) desenvolveram um estudo onde indivíduos da espécie de peixe sablefish (Anoplopoma fimbria) foram expostos a diferentes concentrações de amônia e duas temperaturas: $12{ }^{\circ} \mathrm{C}$ (temperatura da água do mar) e 17 ${ }^{\circ} \mathrm{C}$ (temperatura elevada), por um período de 1 e 2 meses. Os resultados mostraram que a exposição à amônia afetou as respostas antioxidantes, imunológicas não específicas e indicadores de estresse. A atividade da catalase (CAT) e superóxido dismutase (SOD) aumentou, e houve uma diminuição da glutationa reduzida (GSH). Ainda neste trabalho, também houve um aumento na atividade de lisozima, fagocitose, proteína HSP70, cortisol e glicose plasmática. Essas alterações foram marcantes em peixes com exposição à amônia acima de $0,75 \mathrm{mg} / \mathrm{L}$ a $12{ }^{\circ} \mathrm{C}$, e acima de $0,25 \mathrm{mg} / \mathrm{L}$ a $17{ }^{\circ} \mathrm{C}$. Portanto, os resultados indicam que a elevação da temperatura pode aumentar a sensibilidade dos peixes expostos à amônia.

A disponibilidade de oxigênio também pode afetar o funcionamento do organismo dos peixes (Jimenez \& ZaniboniFilho, 2013). Zhao e colaboradores (2020) utilizaram peixes da espécie achigã (Micropterus salmoides) para avaliar diferentes concentrações de amônia combinada à hipóxia. Os autores relataram que a exposição à amônia alterou a atividade de enzimas antioxidantes, metabolismo da glicose, e causou alterações e lesões graves nos tecidos branquiais. Além disso, alterou a expressão de enzimas antioxidantes, proteínas da via apoptótica e inflamatória. As atividades de CAT e SOD variaram em todos os grupos de tratamento; também houve alterações na glicólise anaeróbica (aumento de glicose no sangue, diminuição de glicogênio hepático, acúmulo de lactato e aumento da atividade da lactato desidrogenase). Os resultados indicaram que a exposição combinada gerou efeitos mais deletérios no achigã do que a exposição aos estressores individuais; sendo assim, conclui-se que a hipóxia tem potencial de aumentar a toxicidade da amônia.

A salinidade é considerada um dos fatores ambientais mais importantes que devem ser considerados, pois quando encontrada em altos níveis é capaz de aumentar a tolerância dos peixes à amônia (Kir \& Sunar, 2018; Kir et al., 2019). A dourada do mar (Sparus aurata) e o robalo europeu (Dicentrarchus labrax) são peixes que passam por diferentes salinidades em diferentes estágios da vida (Kir \& Sunar, 2018; Kır et al., 2019). De fato, os níveis de salinidade ideais podem variar de acordo com a espécie e estágio de vida, e precisam ser levados em conta em cada caso particular. Estudos com o peixe dourada do mar avaliando o efeito do nitrogênio amoniacal total (NAT) em diferentes salinidades (10, 20 e 30 ppt), por um período de 24h, 36, 48h e 96h, relataram que os efeitos tóxicos de NAT são 4,5 vezes maiores em salinidade baixa (10 ppt) e 2,2 vezes maiores em salinidade alta (30 ppt). Portanto, os resultados da relação de níveis de salinidade com a amônia testados indicaram que a salinidade tem um efeito protetor, sendo que os peixes da espécie dourada do mar se mostraram mais tolerantes à amônia na mais alta salinidade avaliada pelos autores (Kir \& Sunar, 2018).

Resultado semelhante foi encontrado com o robalo europeu. Kir e colaboradores (2019) também avaliaram o efeito da amônia em diferentes salinidades para os robalos. Foram conduzidos experimentos com diferentes níveis de nitrogênio amoniacal total (NAT) e salinidades distintas durante $24 \mathrm{~h}, 36 \mathrm{~h}, 48 \mathrm{~h}, 96 \mathrm{~h}$. O metabolismo dos peixes foi influenciado pela concentração de amônia, onde concentrações elevadas causaram um aumento no metabolismo, e altos níveis de salinidade testados para os robalos foram capazes de mitigar esses efeitos. Portanto, a exposição combinada a altos níveis de salinidade em robalos europeus foi capaz de aumentar a tolerância à amônia e mitigar os efeitos causados no metabolismo.

A amônia é um poluente tóxico e sua toxicidade pode ser afetada por diferentes fatores ambientais. A elevação da temperatura e da hipóxia possuem efeitos sinérgicos, que aumentaram a toxicidade da amônia. Já a salinidade possui efeito protetor, pois os estudos mostraram que dentro da faixa avaliada, os mais altos níveis de salinidade foram capazes de aumentar a tolerância dos peixes à amônia. No entanto, a toxicidade também é dependente do período de exposição à amônia, onde, períodos mais longos estão relacionados com efeitos mais tóxicos. 


\subsection{Efeitos do nitrito e fatores ambientais que influenciam na sua toxicidade}

O nitrito é tóxico para os peixes mesmo em baixas concentrações (Kubitza, 2019), e pode se acumular nos tecidos por meio da absorção de íons de cloreto do epitélio braquial (Lin et al., 2018). Os efeitos da exposição ao nitrito incluem danos histopatológicos em órgãos-alvos específicos, como hiperplasia e hipertrofia de células epiteliais branquiais, necrose das células hepáticas e atrofia de hepatócito em áreas hemorrágicas no fígado. Além disso, foram observadas patologias associadas ao rim, como lesão do corpúsculo renal, degeneração severa das células dos túbulos renais, hemorragia e difusão dos eritrócitos no líquido intersticial em Oncorhynchus mykiss (Grynevych et al., 2018). Também são relatadas alterações na atividade de enzimas antioxidantes (Lin et al., 2018), no sistema endócrino (Gao et al., 2019) e imunológico nos peixes (Gao et al., 2020).

A temperatura pode influenciar na toxicidade dos compostos, podendo ter efeitos sinérgicos e antagonistas. Peixesgato listrados (Pangasianodon hypophthalmus) foram expostos ao nitrito e a diferentes temperaturas: $27{ }^{\circ} \mathrm{C}$ (temperatura média do local de estudo) e $33{ }^{\circ} \mathrm{C}$ (temperatura elevada prevista), por 7 dias (Ha et al., 2019). Esta combinação foi capaz de aumentar os valores de metahemoglobina no sangue no primeiro dia de experimento, mas os valores diminuíram ao longo do período de exposição. Esse aumento de metahemoglobina foi maior em peixes expostos à temperatura de $27{ }^{\circ} \mathrm{C}$ do que à 33 ${ }^{\circ} \mathrm{C}$, refletindo uma menor absorção de nitrito em temperaturas mais altas. Isso ocorreu devido à capacidade dos peixes-gato listrados de realizarem respiração aérea facultativa, e quando expostos a altas temperaturas reduziram a captação de nitrito pelas brânquias, aumentando a captação de oxigênio através da respiração aérea. Sendo assim, a exposição simultânea ao nitrito e altas temperaturas possuem efeito antagonista.

A salinidade é outro fator ambiental fundamental, pois pode afetar o desenvolvimento e as taxas de sobrevivência dos peixes (Yang \& Chen, 2006). Peixes migratórios foram expostos ao nitrato elevado e diferentes níveis de salinidade, e os resultados mostram que níveis de salinidade mais altos foram capazes de mitigar os efeitos do nitrito em testes de toxicidade aguda (Kir \& Sunar, 2018; Wang et al., 2017). Com o aumento da salinidade nos níveis testados (10, 20 e 30 ppt) o peixe dourada do mar foi mais tolerante ao nitrito, diminuindo os efeitos tóxicos (Kir \& Sunar, 2018). Resultado semelhante foi encontrado em baiacu obscuro (Takifugu obscurus), em que o nitrito foi capaz de diminuir significativamente as taxas de sobrevivência dos peixes juvenis, e o aumento da salinidade mitigou esses efeitos, aumentando as taxas de sobrevivência (Wang et al., 2017).

Altas concentrações de nitrito são frequentemente encontradas em águas doces, salobras e marinhas, e podem se acumular no ambiente de forma natural e serem intensificadas devido as atividades antrópicas. O nitrito é tóxico para os peixes, pois é capaz de converter hemoglobina a metahemoglobina, dificultando a capacidade de oxigênio no sangue, além de reduzir as taxas de sobrevivência nos peixes. Contudo, a exposição simultânea de nitrito e alta temperatura mostrou efeito antagônico, e a combinação de nitrito e altos níveis de salinidade possuem efeito protetor, capazes de mitigar os efeitos tóxicos causado pela exposição ao nitrito.

\subsection{Efeitos do nitrato e fatores ambientais que influenciam na sua toxicidade}

O nitrato entra em organismos aquáticos através da difusão passiva nas células branquiais, e a principal ação tóxica é devido a conversão endógena de nitrato em nitrito, que então causa a formação de metahemoglobina, resultando na perda da capacidade de transporte de oxigênio no sangue (Gomez Isaza et al., 2021) e consequentemente a morte dos indivíduos. Além disso, o nitrato é capaz de interromper a síntese de esteroides, comprometendo a reprodução (Kellock et al., 2018), e causar histopatologias em brânquias de peixes, como necrose, hiperplasia, fusão lamelar, edemas e degeneração da lamela (Paul et al., 2019; Dutra et al., 2019).

O aumento da temperatura pode influenciar na fisiologia dos peixes, com efeitos sinérgicos ou antagonistas. Peixes australianos da espécie Perca prateada, Bidyanus bidyanus, foram expostos ao nitrato em diferentes concentrações e 
temperatura: $28{ }^{\circ} \mathrm{C}$ (temperatura do local de estudo) e $32{ }^{\circ} \mathrm{C}$ (temperatura elevada) por 21 semanas (Gomez Isaza et al., 2021). Os autores relatam que a exposição ao nitrato elevado reduziu a capacidade de transporte de oxigênio no sangue, devido ao aumento da metahemoglobina, em ambas as temperaturas. Já a exposição à temperatura mais alta (32 $\left.{ }^{\circ} \mathrm{C}\right)$ foi capaz de aumentar a área da superfície branquial e a espessura ventricular, independente da concentração do nitrato. Como conclusão, os autores indicam que essa plasticidade do sistema cardiorrespiratório fornece proteção à exposição simultânea ao nitrato e altas temperaturas.

Um estudo realizado com salmonídeo de água doce, Thymallus thymallus, encontrou efeitos antagonistas semelhantes (Opinion et al., 2020). Neste caso, os peixes foram expostos ao nitrato e diferentes temperaturas: $18{ }^{\circ} \mathrm{C}$ (temperatura média da região em estudo) e $22{ }^{\circ} \mathrm{C}$ (temperatura elevada) por 6 semanas. A interação desses dois estressores foi capaz de causar efeitos antagonistas significativos no escopo aeróbico, aumentando a capacidade dos peixes de fornecer oxigênio necessário para as respostas ao estresse, indicando benefícios na tolerância cruzada. Com base nesses resultados é possível entender que a temperatura elevada e exposição ao nitrato é capaz de promover à hipóxia nos peixes pelo baixo transporte de oxigênio. Seguindo a metodologia proposta por Opinion e colaboradores (2020), Rodgers e colaboradores (2021) conduziram um experimento por 8 semanas e avaliaram a hipóxia e tolerância ao calor na mesma espécie Thymallus thymallus. Em altas concentrações de nitrato houve uma diminuição de $1{ }^{\circ} \mathrm{C}$ na tolerância ao calor em peixes expostos a $18{ }^{\circ} \mathrm{C}$, entretanto, em 22 ${ }^{\circ} \mathrm{C}$ foi observado o oposto, houve um aumento de $1{ }^{\circ} \mathrm{C}$. O nitrato também causou alterações histológicas nas brânquias em ambas as temperaturas, como hipertrofia e hiperplasia, que consequentemente diminui a área de superfície respiratória, comprometendo a absorção de oxigênio no organismo. Além disso, a exposição ao nitrato também foi capaz de aumentar significativamente a suscetibilidade à hipóxia em ambas as temperaturas.

O baixo pH é um fator ambiental capaz de aumentar a absorção de alguns poluentes causando o acúmulo desses compostos no fígado e nas brânquias (Çoğun \& Kargin, 2004). Para avaliar como o pH influencia na toxicidade do nitrato, indivíduos de Leiopotherapon unicolor foram expostos simultaneamente ao nitrato e diferentes níveis de $\mathrm{pH}$ ( $\mathrm{pH} 4,0$ ou 7,0) por 28 dias de exposição (Gomez Isaza et al., 2020). Os resultados mostraram que, a exposição ao nitrato levou ao comprometimento da capacidade de transporte de oxigênio, independentes da concentração. Já o efeito combinado do nitrato elevado e baixo $\mathrm{pH}$ agravou os efeitos sobre a capacidade de transporte no sangue, levando a declínios no fitness dos peixes (crescimento, natação e aumento da recuperação pós-exercício).

Para compreender os efeitos da química da água sobre a toxicidade de nitrato, larvas de peixes de três espécies foram expostas ao nitrato e a valores distintos de dureza da água (Moore \& Bringolf, 2020). As concentrações de nitrato variaram de 0 a $4800 \mathrm{mg} / \mathrm{L}$ e três tipos de dureza da água (mole, moderadamente dura e dura) foram testados em larvas de Promelas pimephales, Cyprinella trichroistia e Oreochromis ssp. por 7 dias. Os resultados demonstraram a influência da dureza da água sobre a sensibilidade dos peixes ao nitrato. Larvas de $P$. pimephales foram mais sensíveis ao nitrato em água moderadamente dura e dura, já C. trichroistia foram mais sensíveis em água mole, e Oreochromis ssp. foi a espécie menos sensível, independente da dureza da água. Portanto, a dureza da água é capaz de influenciar na sensibilidade dos peixes ao nitrato.

O nitrato é um poluente onipresente nos ambientes aquáticos e avaliar sua toxicidade diante os fatores que podem influenciá-la é importante para a preservação dos peixes. O nitrato foi capaz de aumentar a suscetibilidade à hipóxia e alterar o sistema cardiovascular, reduzindo a capacidade de transporte de oxigênio, e a interação com baixo pH intensificou esses efeitos levando a declínios no fitness (i.e. aptidão do organismo em termos de crescimento e natação) dos peixes. Já a combinação de nitrato e alta temperatura teve efeitos antagônicos, em que os peixes foram capazes de aumentar a capacidade de fornecer oxigênio. A dureza da água também pode influenciar na toxicidade do nitrato, aumentando ou diminuindo a sensibilidade de larvas de peixes, no entanto, os efeitos são dependentes da espécie em estudo. 


\section{Considerações Finais}

Os parâmetros de qualidade da água influenciam na toxicidade dos compostos nitrogenados (nitrito, nitrato e amônia), podendo ter efeitos sinérgicos, intensificando os efeitos tóxicos, ou antagonistas, quando o efeito de dois estressores é menor que do estressor individual. A relação dos parâmetros físico-químicos da água e a toxicidade dos compostos nitrogenados é representado na Figura 2. A hipóxia e o baixo pH mostraram efeitos sinérgicos, em que foram capazes de aumentar a toxicidade da amônia e nitrato. Já altas salinidades mitigaram os efeitos da exposição à amônia e nitrito e, portanto, possuem efeito protetor. $\mathrm{O}$ aumento da temperatura causou efeitos distintos dentre os compostos nitrogenados, com efeitos sinérgicos quando os peixes foram expostos simultaneamente à amônia, e efeitos antagônicos quando expostos ao nitrito e nitrato. Variações na dureza da água também influenciaram na sensibilidade dos peixes ao nitrato, sendo dependente da espécie em estudo. Portanto, essa revisão evidenciou a necessidade da avaliação da toxicidade dos compostos nitrogenados em peixes combinado a outras condições ambientais, nesse caso os parâmetros físico-químicos da água: pH, temperatura da água, salinidade, oxigênio dissolvido e dureza da água.

Estudos futuros devem seguir avaliando o cenário de mudanças globais com alterações no clima (aumento de temperatura e mudança no regime pluvial) e no uso do solo (aumento de área com agricultura intensiva) em associação com os efeitos nos animais. Assim, estas pesquisas devem entender e/ou buscar formas de evitar consequências graves das ações antrópicas desordenadas como baixa qualidade de vida e até as extinções de espécies e assim impedir a perda de biodiversidade.

Figura 2. Compostos nitrogenados e influência dos parâmetros físico-químicos em sua toxicidade.
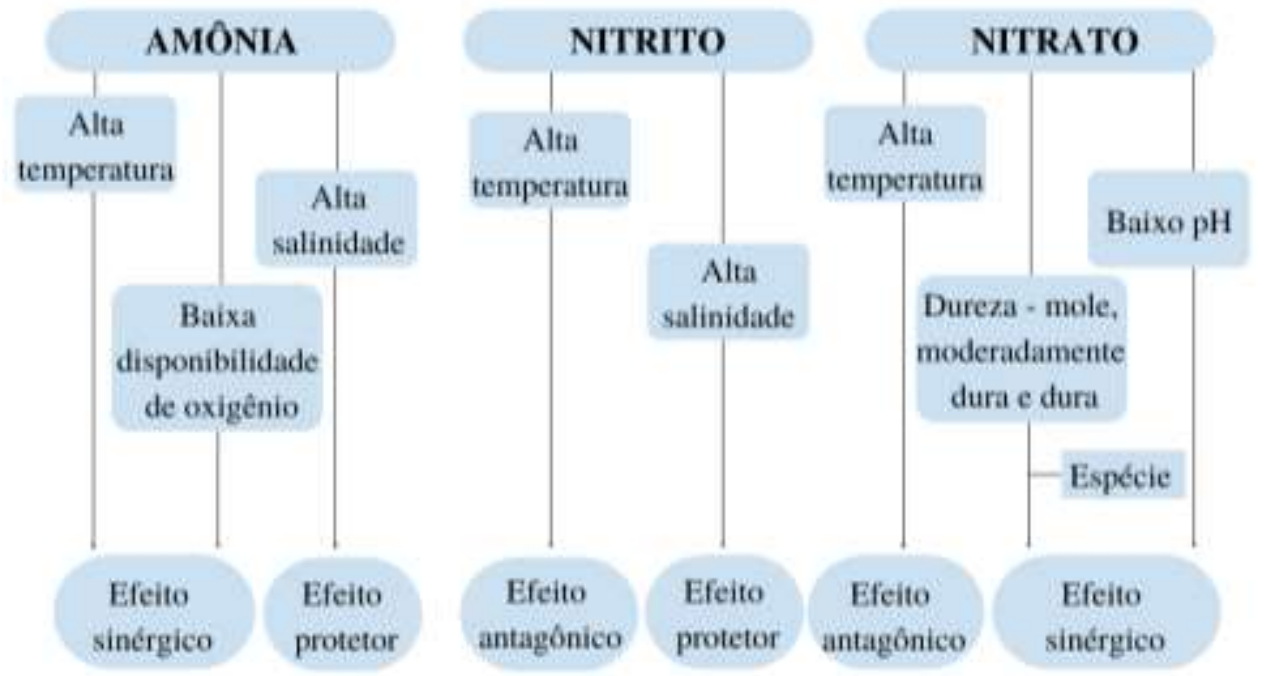

Fonte: Autores (2021).

\section{Agradecimentos}

Os autores agradecem à Universidade Federal de São João del-Rei campus Centro-Oeste Dona Lindu pelo apoio e suporte. Este estudo foi financiado pelo Projeto de Pesquisa Ecológica de Longo Prazo (PELD), site-4 (CNPq / CAPES / FAPEMIG - Proc. $\mathrm{N}^{\circ} 441481$ / 2016-7). Além disso, este estudo recebeu suporte financeiro da Coordenação de Aperfeiçoamento de Pessoal de Nível Superior - Brasil (CAPES) - (subsídio número 88887.124083 / 2016-00) e Código Financeiro 001. 


\section{Referências}

Alfonso, S., Gesto, M., \& Sadoul, B. (2020). Temperature increase and its effects on fish stress physiology in the context of global warming. Journal of Fish Biology, 39. https://doi.org/10.1111/jfb.14599

Côté, I. M., Darling, E. S., \& Brown, C. J. (2016). Interactions among ecosystem stressors and their importance in conservation. Proceedings of the Royal Society B: Biological Sciences, 283(1824). https://doi.org/10.1098/rspb.2015.2592

Çoğun, H. Y., \& Kargin, F. (2004). Effects of pH on the mortality and accumulation of copper in tissues of Oreochromis niloticus. Chemosphere, 55(2), 277282. https://doi.org/10.1016/j.chemosphere.2003.10.007

Dutra, F. M., Cidemar Alab, J. H., Costa Gomes, M. K., Furtado, P. S., Valenti, W. C., \& Cupertino Ballester, E. L. (2019). Nitrate acute toxicity to post larvae and juveniles of Macrobrachium amazonicum (Heller, 1862). Chemosphere, 242, 125229. https://doi.org/10.1016/j.chemosphere.2019.125229

Ferrari, R. (2015). Writing narrative style literature reviews. Medical Writing, 24(4), 230-235. https://doi.org/10.1179/2047480615z.000000000329

Gao, X. Q., Fei, F., Huo, H. H., Huang, B., Meng, X. S., Zhang, T., \& Liu, B. L. (2020). Impact of nitrite exposure on plasma biochemical parameters and immune-related responses in Takifugu rubripes. Aquatic Toxicology, 218, 105362. https://doi.org/10.1016/j.aquatox.2019.105362

Gao, X. Q., Fei, F., Huo, H. H., Huang, B., Meng, X. S., Zhang, T., Liu, W. Bin, \& Liu, B. L. (2019). Exposure to nitrite alters thyroid hormone levels and morphology in Takifugu rubripes. Comparative Biochemistry and Physiology Part - C: Toxicology and Pharmacology, $225,108578$. https://doi.org/10.1016/j.cbpc.2019.108578

Gomez Isaza, D. F., Cramp, R. L., \& Franklin, C. E. (2020). Simultaneous exposure to nitrate and low pH reduces the blood oxygen-carrying capacity and functional performance of a freshwater fish. Conservation Physiology, 8(1), 1-15. https://doi.org/10.1093/conphys/coz092

Gomez Isaza, D. F., Cramp, R. L., \& Franklin, C. E. (2021). Thermal plasticity of the cardiorespiratory system provides cross-tolerance protection to fish exposed to elevated nitrate. Comparative Biochemistry and Physiology Part - C: Toxicology and Pharmacology, $240,108920$. https://doi.org/10.1016/j.cbpc.2020.108920

Grynevych, N., Sliusarenko, A., Dyman, T., Sliusarenko, S., Gutyj, B., \& Kukhtyn, M. (2018). Impact of provincial water management on environment and social welfare in West of Zayanderood Basin, Iran. Ukrainian Journal of Ecology, 8(1), 41-50. https://doi.org/10.15421/2018

Ha, N. T. K., Huong, D. T. T., Phuong, N. T., Bayley, M., \& Jensen, F. B. (2019). Impact and tissue metabolism of nitrite at two acclimation temperatures in striped catfish (Pangasianodon hypophthalmus). Aquatic Toxicology, 212, 154-161. https://doi.org/10.1016/j.aquatox.2019.05.008

Jimenez, J. E., \& Zaniboni-Filho, E. (2013). Adaptations of the piava (Leporinus obtusidens) juvenile exposed to hypoxia. Boletim Do Instituto de Pesca, $39(4), 439-444$.

Kellock, K. A., Moore, A. P., \& Bringolf, R. B. (2018). Chronic nitrate exposure alters reproductive physiology in fathead minnows. Environmental Pollution, 232, 322-328. https://doi.org/10.1016/j.envpol.2017.08.004

Kim, J. H., \& Kang, J. C. (2016). The immune responses in juvenile rockfish, Sebastes schlegelii for the stress by the exposure to the dietary lead (II). Environmental Toxicology and Pharmacology, 46, 211-216. https://doi.org/10.1016/j.etap.2016.07.022

Kim, J. H., Park, H. J., Hwang, I. K., Han, J. M., Kim, D. H., Oh, C. W., Lee, J. S., \& Kang, J. C. (2017). Toxic effects of juvenile sablefish, Anoplopoma fimbria by ammonia exposure at different water temperature. Environmental Toxicology and Pharmacology, 54, 169-176. https://doi.org/10.1016/j.etap.2017.07.008

Kir, M., \& Sunar, M. C. (2018). Acute Toxicity of Ammonia and Nitrite to Sea Bream, Sparus aurata (Linnaeus, 1758), in Relation to Salinity. Journal of the World Aquaculture Society, 49(3), 516-522. https://doi.org/10.1111/jwas.12448

Kır, M., Sunar, M. C., \& Gök, M. G. (2019). Acute ammonia toxicity and the interactive effects of ammonia and salinity on the standard metabolism of European sea bass (Dicentrarchus labrax). Aquaculture, 511, 734273. https://doi.org/10.1016/j.aquaculture.2019.734273

Kubitza, F. (2019). O impacto da amônia, do nitrito e do nitrato sobre o desempenho e a saúde dos peixes e camarões. A água na aquicultura | Parte 3. 1-27.

Lefevre, S., McKenzie, D. J., \& Nilsson, G. E. (2017). Models projecting the fate of fish populations under climate change need to be based on valid physiological mechanisms. Global Change Biology, 23(9), 3449-3459. https://doi.org/10.1111/gcb.13652

Li, M., Zhang, M., Qian, Y., Shi, G., \& Wang, R. (2020). Ammonia toxicity in the yellow catfish (Pelteobagrus fulvidraco): The mechanistic insight from physiological detoxification to poisoning. Fish and Shellfish Immunology, 102, 195-202. https://doi.org/10.1016/j.fsi.2020.04.042

Lin, Y., Miao, L. H., Pan, W. J., Huang, X., Dengu, J. M., Zhang, W. X., Ge, X. P., Liu, B., Ren, M. C., Zhou, Q. L., Xie, J., Pan, L. kun, \& Xi, B. wen. (2018). Effect of nitrite exposure on the antioxidant enzymes and glutathione system in the liver of bighead carp, Aristichthys nobilis. Fish and Shellfish Immunology, 76, 126-132. https://doi.org/10.1016/j.fsi.2018.02.015

Martinez, C. B. R., Azebedo, F., \& Winkaler, E. U. (2006). Toxicidade e Efeitos da Amônia em Peixes Neotropicais. Tópicos Especiais Em Biologia Aquática e Aqüicultura, 81-95.

Mehta, K. (2017). Impact of Temperature on Contaminants Toxicity in Fish Fauna: A Review. Indian Journal of Science and Technology, 10(18), 1-6. https://doi.org/10.17485/ijst/2017/v10i18/112663

Moore, A. P., \& Bringolf, R. B. (2020). Comparative Toxicity of Nitrate to Common and Imperiled Freshwater Mussel Glochidia and Larval Fishes. Archives of Environmental Contamination and Toxicology, 78(4), 536-544. https://doi.org/10.1007/s00244-020-00708-z 
Research, Society and Development, v. 10, n. 11, e359101119779, 2021

(CC BY 4.0) | ISSN 2525-3409 | DOI: http://dx.doi.org/10.33448/rsd-v10i11.19779

Opinion, A. G. R., De Boeck, G., \& Rodgers, E. M. (2020). Synergism between elevated temperature and nitrate: Impact on aerobic capacity of European grayling, Thymallus thymallus in warm, eutrophic waters. Aquatic Toxicology, 226, 105563. https://doi.org/10.1016/j.aquatox.2020.105563

Pankhurst, N. W., \& Munday, P. L. (2011). Effects of climate change on fish reproduction and early life history stages. Marine and Freshwater Research, 62(9), 1015-1026. https://doi.org/10.1071/MF10269

Paul, S., Mandal, A., Bhattacharjee, P., Chakraborty, S., Paul, R., \& Kumar Mukhopadhyay, B. (2019). Evaluation of water quality and toxicity after exposure of lead nitrate in fresh water fish, major source of water pollution. Egyptian Journal of Aquatic Research, 45(4), 345-351. https://doi.org/10.1016/j.ejar.2019.09.001

Randall, D. J., \& Tsui, T. K. N. (2002). Ammonia toxicity in fish. Comparative Biochemistry and Physiology Part A: Molecular \& Integrative Physiology, 124, S62. https://doi.org/10.1016/s1095-6433(99)90247-7

Rodgers, E. M., Opinion, A. G. R., Gomez Isaza, D. F., Rašković, B., Poleksić, V., \& De Boeck, G. (2021). Double whammy: Nitrate pollution heightens susceptibility to both hypoxia and heat in a freshwater salmonid. Science of the Total Environment, 765. https://doi.org/10.1016/j.scitotenv.2020.142777

Silva, M. J. Dos S., Costa F. B. C., Leme, F. P.; Takata, R., Costa, D. C., Matitioli, C. C., Luz, R. K., Miranda-Filho, K. C. (2017). Biological responses of Neotropical freshwater fish Lophiosilurus alexandri exposed to ammonia and nitrite. Science of the Total Environment, 616-617, 1566-1575.

Vitousek, P. M., Mooney, H. A., Lubchenco, J., Melillo, J. M. (1997). Human Domination of Earth Ecosystems. Science, 278(5335), 494-499.

Wang, J., Tang, H., Zhang, X., Xue, X., Zhu, X., Chen, Y., \& Yang, Z. (2017). Mitigation of nitrite toxicity by increased salinity is associated with multiple physiological responses: A case study using an economically important model species, the juvenile obscure puffer (Takifugu obscurus). Environmental Pollution, 232, 137-145. https://doi.org/10.1016/j.envpol.2017.09.026

Wang, Y., Kong, X., Peng, Z., Zhang, H., Hu, W., \& Zhou, X. (2020) Retenção de nitrogênio e fósforo no Lago Chaohu, China: implicações para o manejo da eutrofização. Environmental Science and Pollution Research. 27, 41488-41502. https://doi.org/10.1007/s11356-020-10024-7

Yang, Z., \& Chen, Y. (2006). Salinity tolerance of embryos of obscure puffer Takifugu obscurus. Aquaculture, 253(1-4), 393-397. https://doi.org/10.1016/j.aquaculture.2005.08.014

Zhang, W., Xia, S., Zhu, J., Miao, L., Ren, M., Lin, Y., Ge, X., \& Sun, S. (2019). Growth performance, physiological response and histology changes of juvenile blunt snout bream, Megalobrama amblycephala exposed to chronic ammonia. Aquaculture, 506, 424-436. https://doi.org/10.1016/j.aquaculture.2019.03.072

Zhao, L., Cui, C., Liu, Q., Sun, J., He, K., Adam, A. A., Luo, J., Li, Z., Wang, Y., \& Yang, S. (2020). Combined exposure to hypoxia and ammonia aggravated biological effects on glucose metabolism, oxidative stress, inflammation and apoptosis in largemouth bass (Micropterus salmoides). Aquatic Toxicology, 224, 105514. https://doi.org/10.1016/j.aquatox.2020.105514 